\title{
Recent Advances in Microstructured Fibers for Laser Delivery and Generation
}

\author{
J.R. Hayes ${ }^{1}$, M.N. Petrovich ${ }^{1}$, F. Poletti ${ }^{1}$, P. Horak $^{1}$, N.G.R. Broderick ${ }^{1}$, Xian Feng ${ }^{1}$, S.X. \\ Dasgupta $^{1}$, Wei Loh ${ }^{1}$, Debashri Ghosh, ${ }^{2}$ Mrinmay Pal, ${ }^{2}$ Shyamal K. Bhadra, ${ }^{2}$ K.K. Chen ${ }^{1}$, J.H.V. \\ Price $^{1}$, S.U. Alam ${ }^{1}$ and D.J. Richardson ${ }^{1}$. \\ ${ }^{1}$ Optoelectronics Research Centre, University of Southampton, Southampton, SO17 1BJ, UK \\ ${ }^{2}$ Optics Laboratory, Central Glass \& Ceramic Research Institute Kolkata, CSIR, India \\ djr@orc.soton.ac.uk
}

\begin{abstract}
We report recent advances in the development of fibers for the delivery and generation of both single-mode and heavily multimode laser beams as well as recent progress in fibers for supercontinuum generation in spectral regimes spanning the visible to mid-IR.
\end{abstract}

\section{INTRODUCTION}

Microstructured optical fiber (MOF) technology provides a powerful means to develop fibers with unique and enabling properties with potential uses spanning a very wide range of applications. Of all the opportunities provided by MOF technology amongst the most novel and intriguing are: (a) the possibility to guide light in air (through photonic band-gap or anti-resonance based effects); (b) single mode guidance over extended spectral ranges (referred to as endlessly single mode (ESM) guidance); and (c) the fabrication of fibers with very much larger values of Numerical Aperture (NA) than possible using conventional fiber fabrication approaches. It is also important to realize that the MOF technology allows the fabrication of fibers using a single material - considerably enhancing the ease of producing fibers in new materials, such as polymers and compound glasses. This offers access to material properties that are substantially different to those of silica based glasses allowing, for example, fibers with transmission extending into the mid-IR, or ultrahigh values of nonlinearity per unit length. In the following sections we review recent progress in the design and fabrication of fibers that exploit several of these opportunities for the delivery and generation of high power laser radiation and/or broadband light in different spectral regimes.

\section{PHOTONIC BAND GAP FIBERS}

The primary benefits of photonic band gap fibers for power delivery relate to the low nonlinearity associated with the air-core guidance and the prospects for reduced losses in wavelength regimes in which the inherent glass loss would make solid-core variants unusable. Minimizing the modal overlap with the solid regions of the fiber is critical to optimizing the performance of such fibers from these perspectives. Moreover, in many applications, particularly those involving short pulses and/or relatively short delivery lengths, maintaining single transverse mode guidance is a key issue, as is maintaining the maximum usable bandwidth. With this in mind we have conducted extensive numerical and theoretical studies to identify fiber designs providing robust, broadband guidance [1-3]. In Figure 1 we show two fibers fabricated in our facilities with a suitable choice of geometry according to the design rules derived. These pictures show fibers with two sizes of defect created by the omission of 3 and 19 central capillaries. Measurements confirm the broad usable bandwidths and show that the effective modal overlap with the solid regions of the fiber decreases steadily with increasing core size as predicted [4]. It is however to be appreciated that this is associated with a corresponding increase in the number of modes supported by the structure. Only by going to the smallest scale core is robust single mode guidance achievable, as reported in [4]. Exploiting the low overlap factors associated with 19-cell designs we have developed fibers operating at wavelengths $>2.5 \mu \mathrm{m}$, well beyond the accepted transparency window of silica [2]. 


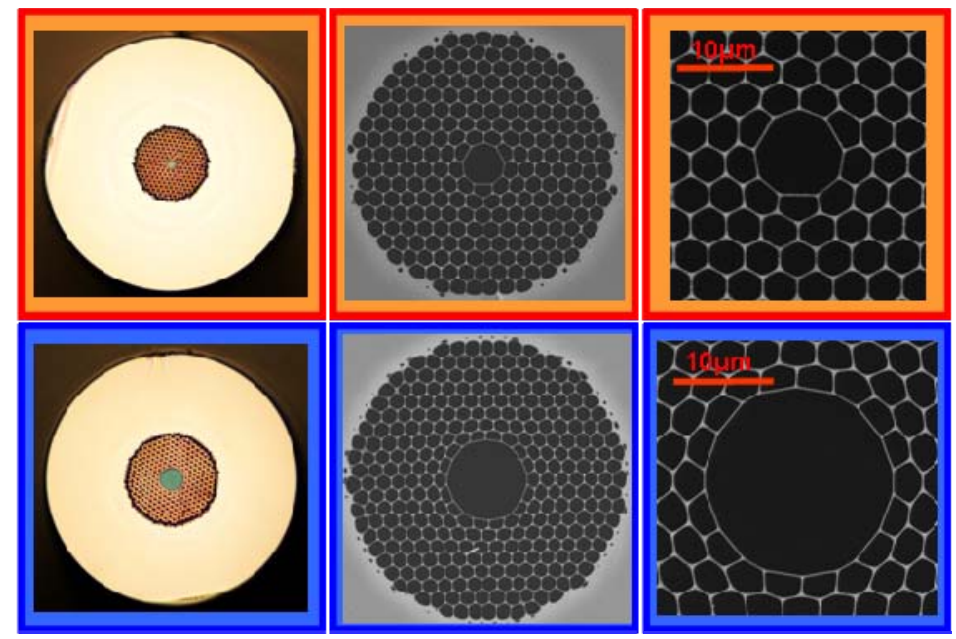

Figure 1. (Top) single mode PBG fiber incorporating a 3-cell core; (Bottom) 19-cell PBGF for extended near IR broadband transmission.

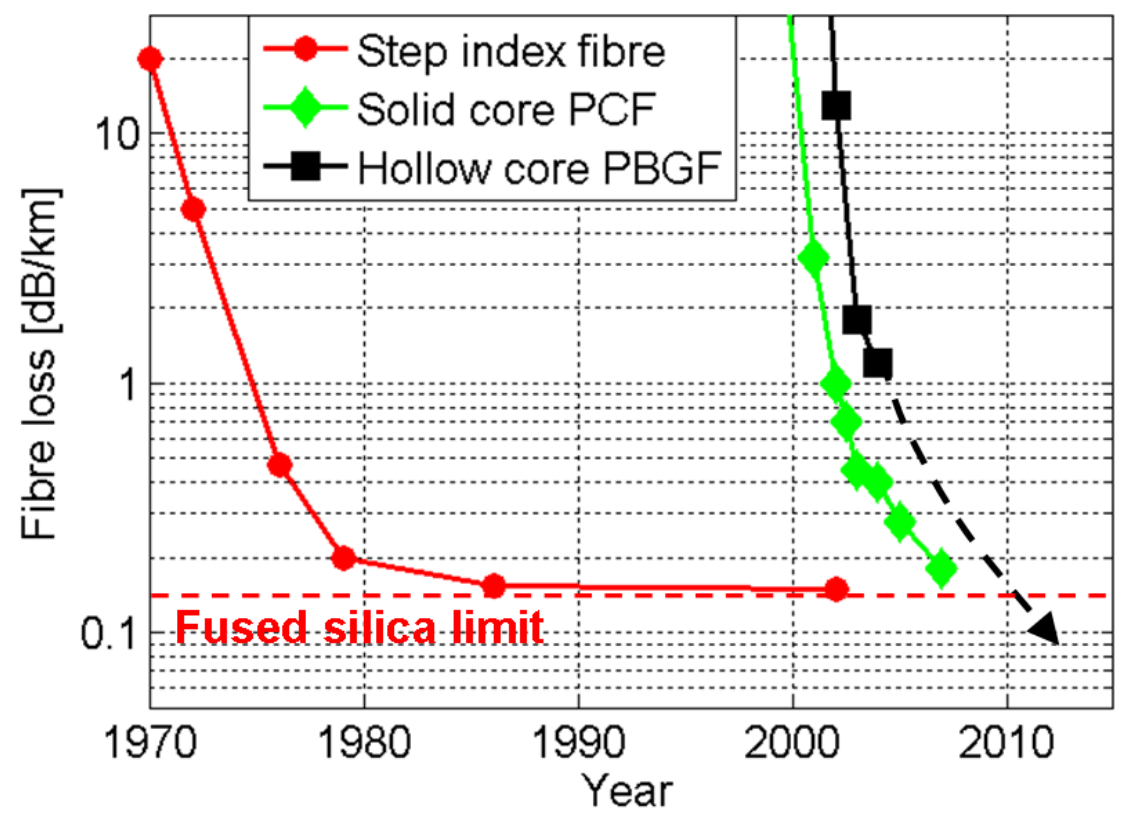

Figure 2. Minimum loss characteristics of both solid and hollow core fibers as a function of year charting the astounding progress made in fiber fabrication procedures.

It is interesting to speculate as to the ultimate loss levels achievable within PBGFs. Impressive improvements in the design and fabrication techniques have reduced the loss in these fibers from several $\mathrm{dB} / \mathrm{m}$ to $1.2 \mathrm{~dB} / \mathrm{km}$ at $1600 \mathrm{~nm}[5] \mathrm{in}$ the space of only a few years (square markers in Figure 2). Although this result is an outstanding achievement in itself, this loss level is still nearly an order of magnitude higher than the standard single mode fiber (SSMF) level. It is worth stressing though that this loss difference is simply due to the different technological maturity between the two fiber types and, relaxing the requirements for single mode operation, no fundamental physical limit is currently identified which should prevent PBGFs from achieving a loss lower than the intrinsic limit of solid fibers based on fused/doped silica. 
Since in PBGFs the fraction of optical power guided in air is ranges from 97 to $>99 \%$, the Rayleigh scattering which dominates losses in conventional SSMF is typically negligible, and therefore losses in these air core fibers are fundamentally dominated by two mechanisms:

(1) Surface scattering at the core glass-air interface and

(2) Infrared phononic absorptions in the glass matrix.

The first mechanism occurs due to the effects of residual imperfections [6], or intrinsic thermodynamic fluctuations (surface capillary waves) which are frozen-in during glass solidification [5]. The impact can be significantly reduced by improving the fabrication process and tailoring the glass properties - hence reducing the overall surface roughness - and through careful fiber design aimed at reducing the amount of optical power at the glass-air interface. The simplest approach to address the latter is to increase the core radius $\mathrm{R}$ by making the core defect larger. As shown in the simulation results detailed in Figure 3, the surface scattering is roughly proportional to $\mathrm{R}^{-3}$ and therefore going from a conventional 7 cell defect core PBGF (7c) to a 61 cell defect core one (61c) could decrease losses by a factor of $\sim 20$. Of course the number of guided modes increases quadratically with $\mathrm{R}$ and therefore this will be accompanied by some degree of multimodality which may need to be managed for some applications. A second solution is to increase the air filling fraction to achieve a larger hole separation and core size for the same central PBG wavelength. This also results in a wider PBG bandwidth. Surface roughness reduction can be achieved by improving the fabrication procedures, using different glass choices and surface treatments [7] and reducing the thermal history of the glass. Using similar approaches the losses of solid core photonic crystal fibers, which also suffer from surface scattering, have been reduced to 0.18 $\mathrm{dB} / \mathrm{km}[8,9]$, very close to the fundamental glass limit (see Figure 2).

The second loss mechanism can be effectively addressed by reducing the amount of power in the glass for example again by increasing the core radius and the air-filling fraction in the periodic cladding. The actual loss minimum occurs where the scattering and infrared absorption contributions are approximately equal. Since the scattering mechanisms detailed above have an inverse cubic dependence on wavelength, and only a fraction of one percent of the power is guided in the glass, it is anticipated that the loss minimum will lie somewhere between 1.8 and $2.1 \mu \mathrm{m}[5,6]$.
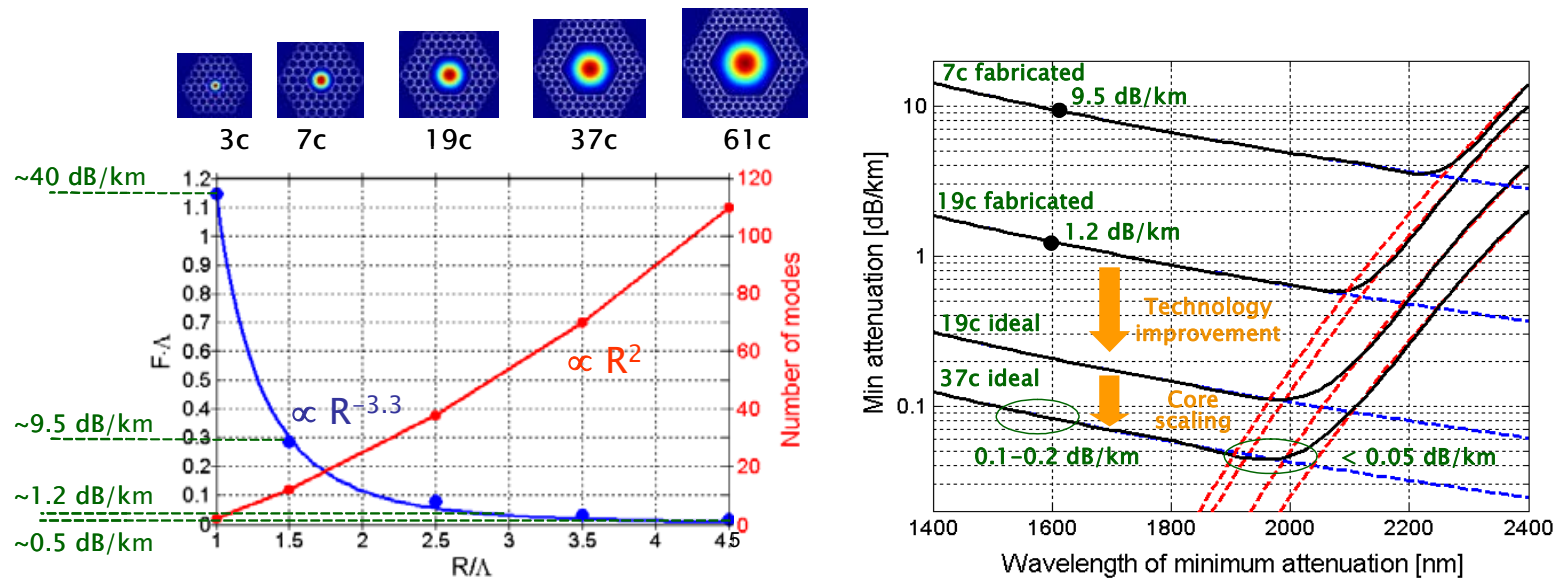

Figure 3. Left - The variation of loss at $1550 \mathrm{~nm}$ and the number of modes versus core design. The parameter $\mathrm{F}$ is directly related to the loss coefficient as indicated. Right - Loss versus wavelength for different core designs.

Using the above approaches, we believe that although challenging it is reasonable to expect a realistic loss below 0.1 $\mathrm{dB} / \mathrm{km}$, and possibly in the region of $0.05 \mathrm{~dB} / \mathrm{km}$, with an overall 20 times improvement over the current $1.2 \mathrm{~dB} / \mathrm{km}$ record achieved in a 19 cell core fiber (where $2 \mathrm{x}$ improvement will come from surface roughness reduction, $2 \mathrm{x}$ from a higher air filling fraction, $2.5 \mathrm{x}$ from core enlargement and $2 \mathrm{x}$ from the wavelength shift). These fibers are also expected to have a nonlinear coefficient extremely close to that of air $\left(\gamma \sim 0.001 \mathrm{~W}^{-1} \mathrm{~km}^{-1}[10]\right)$, nearly three order of magnitude lower than in conventional telecoms fibers, and a bandwidth of several hundreds of nanometres. It is thus not implausible that such fibers could even ultimately be considered for telecommunications applications. 


\section{HIGH NA JAC FIBERS}

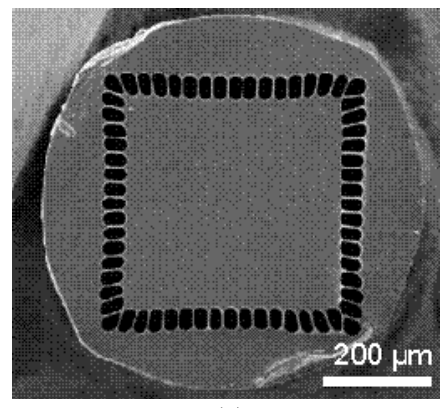

(a)

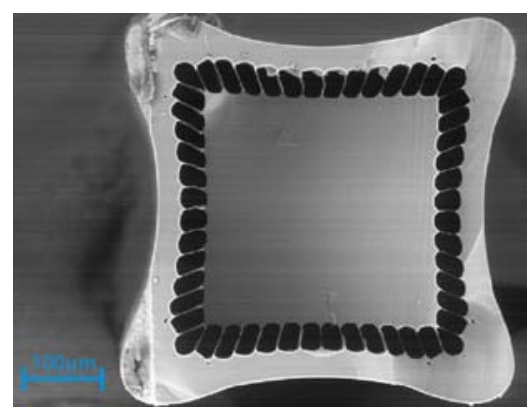

(b)

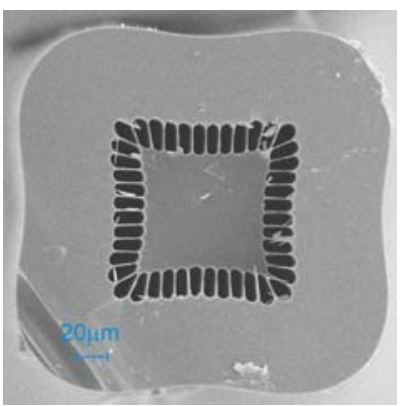

(c)

Figure 4. (a) \& (b) Large core square JAC fibers for high power diode laser delivery, and (c) an active Yb-doped JAC fiber with small core-to-cladding area ratio for pulsed laser applications.

High-NA, large core Jacketed Air Clad (JAC) fibers are of relevance to a range of applications most notably in the context of fiber lasers [11][12] and multimode laser diode beam delivery since they allow confinement of the guided light to a far smaller cross-sectional area than possible in either all-glass, or polymer-clad structures. This tighter optical confinement can be used to make shorter fiber amplifier and laser devices and thereby reducing the impact of fiber nonlinearities and/or dispersion. Numerical apertures approaching unity are now possible [13]. In addition, the technology also allows for the ready implementation of fibers with different cross-sectional profiles. For example, in Figure 4(a) we show an SEM of a square JAC fiber recently developed at Southampton for high power laser beam delivery in which the square near field profile is used to provide benefit in terms of pixel-definition in applications such as flat screen display manufacture [14], amongst others. In Fig 4(c) we show an SEM image of an active Yb-doped JAC fiber with a small cladding-to-active core area ratio and with a Large Mode Area core allowing for short amplifier device lengths as required to minimise nonlinear effects in high peak power pulsed fiber laser systems.

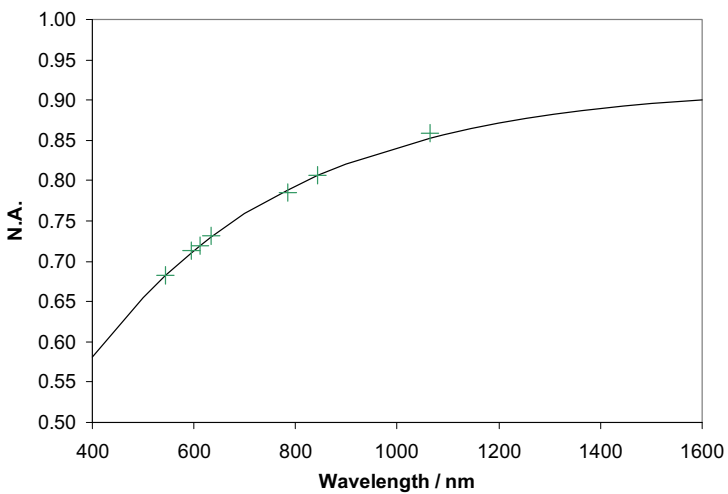

Figure 5. Measured NA of the fiber shown in Fig4(c) as a function of wavelength.

To illustrate the high NAs achievable in Figure 5 we plot the results of an NA measurement on the fiber shown in Figure 4(c) where it is seen that as a result of the very thin struts ( $220 \mathrm{~nm}$ width) and high air fill fractions in the fiber cladding we obtain an NA approaching 0.85 at $980 \mathrm{~nm}$ - the pump wavelength of choice for this active fiber. To put this in perspective the NA of most polymer dual-clad fibers used in high power fiber lasers is around 0.44.

The fiber shown in Fig 4(b) has an NA of 0.61 at $808 \mathrm{~nm}$, its design wavelength, and is distinguished by the combination of the size of the inner cladding and high NA which are essential in order to scale the technology to accommodate beams at the multi-kW level. In order to achieve this combination of fiber properties it is necessary to accurately control the relative feature size of the struts and inner cladding which differ by about three orders of magnitude and which is 
extremely challenging from a fabrication perspective. Note that in developing this technology we have successfully worked with OpTek Ltd to develop laser based techniques to prepare the end-facets to facilitate beam coupling and splicing of these new fiber types.

\section{LARGE MODE AREA ENDLESSLY SINGLE MODE FIBERS FOR MID-IR BEAM DELIVERY AND SUPERCONTINUUM GENERATION}

Endlessly single mode (ESM) fibers are of interest for a range of potential applications and hexagonally stacked fibers with differing core structure have been developed and characterized in terms of their modedness, effective area, and bend-loss characteristics. In many single wavelength applications conventional doped fiber technology can arguably deliver fibers capable of similar performance. However, MOF technology offers potential advantage, when single mode guidance is required over extended wavelength ranges. For example, we have recently developed structures capable of delivering both fundamental and second harmonic radiation in a single mode from a pulsed Q-switched Nd:YAG laser as required for various direct-write materials processing applications [15].
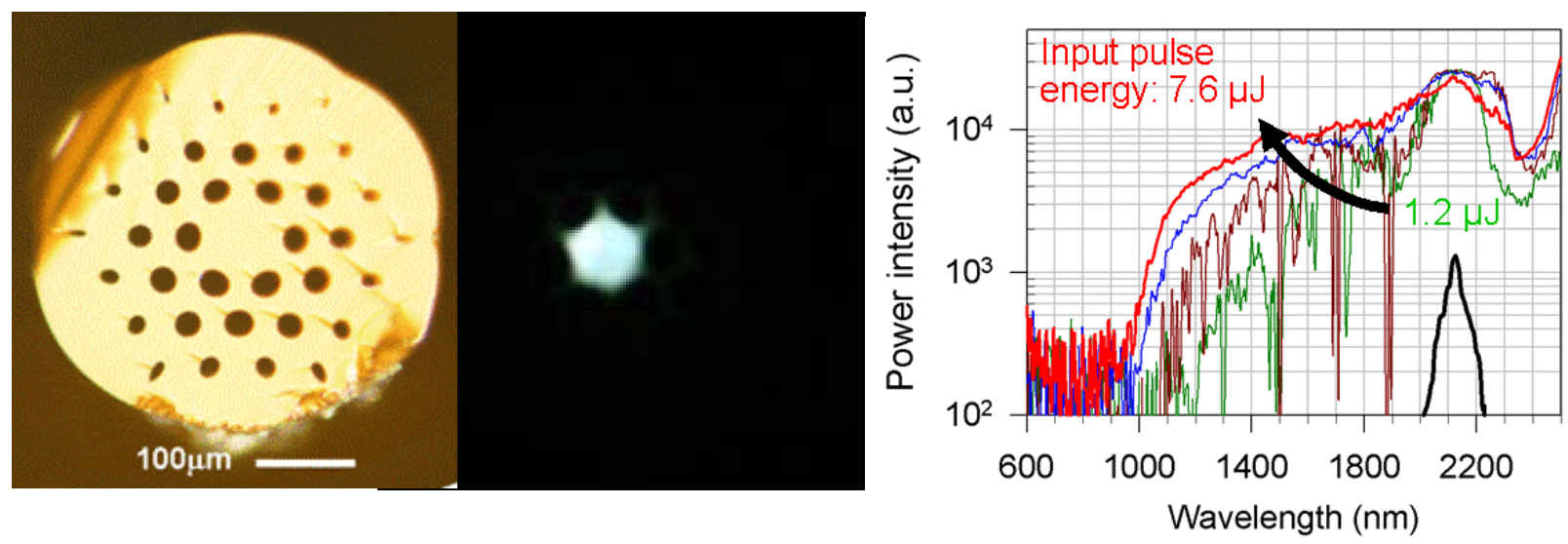

Figure 6. Left - LMA Tellurite fiber with an effective area of $\sim 3000 \mu \mathrm{m}^{2}$ : Centre - mode profile : Right - Supercontiuum spectrum.

In doing this work we identified additional parasitic modes guided due to bandgap/low density of states effects capable of degrading the mode quality and which may limit the utility of such fibers in certain instances [16]. It is to be appreciated that the use of ESM fibers in additional wavelength regimes has also become the focus of considerable attention with the first reports of such fibers being made in new materials such as Tellurite glass (transparent at wavelengths up to $5 \mu \mathrm{m}$ ) [17] and in silver halides (capable of guiding light at wavelength beyond $20 \mu \mathrm{m}$ ) [18]. As well as being of interest for high power laser delivery, such fibers are of use in applications such as modal filtering in spaceborne astronomy [19], and broadband mid-IR supercontinuum generation where the large effective area is helpful since it allows SC generation at high pulse energies [20]. This is important since the pulse energies achievable in small core soft glass fiber variants and not sufficient for many applications due the high nonlinearities per unit length and practical power handling thresholds. For example, the Tellurite fiber shown in Figure 6 was used to demonstrate SC generation using high energy $(>15 \mu \mathrm{J}) 120$ fs-pulses at 1550nm [21] - the corresponding spectrum and mode-profile confirming single mode operation are also shown in Figure 6. 


\section{SMALL CORE FIBERS FOR HIGH SPECTRAL DENSITY VISIBLE SUPERCONTINUUM GENERATION}

As a final demonstration of the significance of microstructured fiber technology in the field of lasers we finish by reporting the design and fabrication of a microstructured fiber optimized to produce high spectral density supercontimuum in the visible region of the spectrum. For an excellent review of supercontinuum generation in fibers please refer to reference [21].

Our work was motivated by a recent study which showed that a high air-fill-fraction PCF design with a core diameter in the $4.2 \mu \mathrm{m}-4.7 \mu \mathrm{m}$ range produced more continuum in the $400-450 \mathrm{~nm}$ wavelength range than low air-fill designs such as endlessly single mode fibers [22]. Following this observation, and noting that the larger core size should allow for higher coupling efficiency from LMA fiber based pulsed sources and improved power handling capability, we developed a $4.4 \mu \mathrm{m}$ core diameter, high-delta PCF for high average power continuum generation [23]. The large core diameter led to an $80 \%$ coupling efficiency from a recently developed high power picosecond fiber MOPA operating at average powers of up to $100 \mathrm{~W}$ and allowed a significant improvement in optical-to-optical conversion efficiency to be demonstrated as compared to visible continuum generation results from CW pumped sources [24].

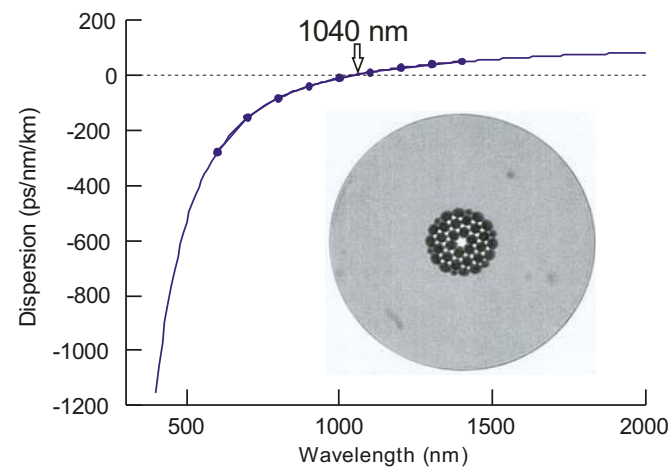

Figure 7(a ). Measured dispersion data (blue dots), extrapolated dispersion curve (solid line) and the corresponding fiber structure

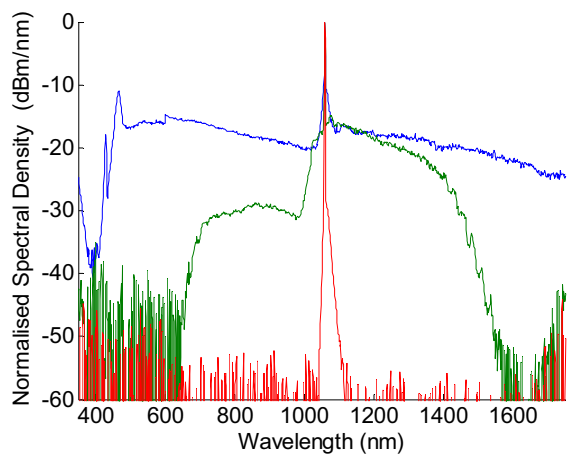

Figure 7 (c). Supercontinuum evolution in a $2 \mathrm{~m}$ long PCF at $0.15 \mathrm{~W}, 11 \mathrm{~W}$ and $57 \mathrm{~W}$ of incident pump power

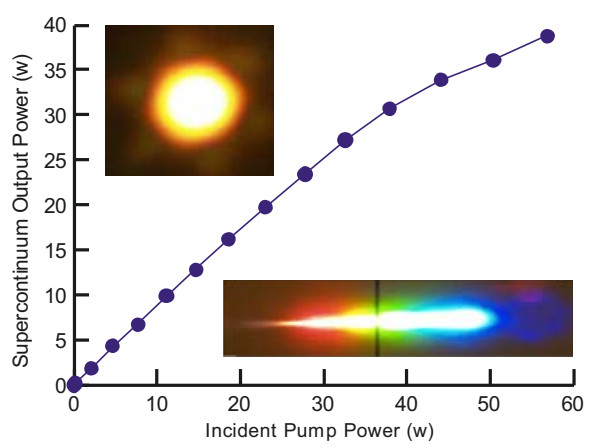

Figure 7(b). Supercontinuum output power vs incident power. Inset show the far field pattern of the output beam and prism separated white light

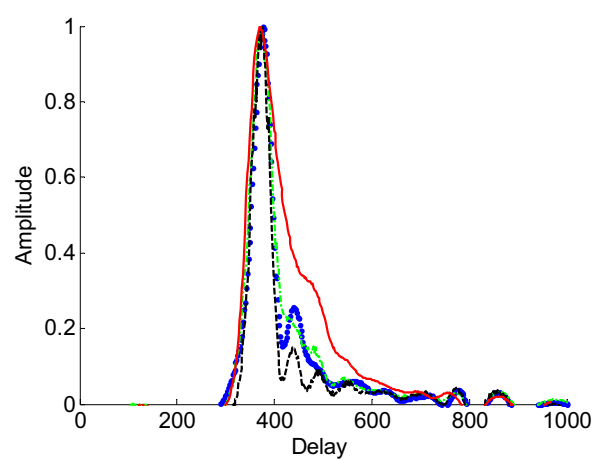

Figure 7(d). Pulse shape of transmitted low power pump (blue dotted line), filtered at $1186.6 \mathrm{~nm}$ (green dash dotted line), filtered at $1317.6 \mathrm{~nm}$ (black dashed line) and broadband (red solid line).

A picture of the fiber is shown in Figure 7(a). In Figure 7(b) we plot the output supercontinuum power as a function of incident pump power (pulse duration $=20 \mathrm{ps}$, pulse repetition rate $=118 \mathrm{MHz}$ ) where it is seen that output powers of up to $39 \mathrm{~W}$ are achieved using a $2 \mathrm{~m}$ length of fiber for the maximum usable incident pump power of $57 \mathrm{~W}$. Inset we show image of the output mode and the output beam after being frequency dispersed from a bulk grating. In Figure 7(c) we plot the optical spectrum at the fiber output where it is seen that the spectral output spans the $0.4-1.7 \mu \mathrm{m}$ wavelength range - more recent measurements actually show the spectrum to extend out to $2.25 \mu \mathrm{m}$. A high and relatively uniform 
spectral power density is achieved across the visible spectral region. A record spectral density of $\sim 31.7 \mathrm{~mW} / \mathrm{nm}$, corresponding to peak power density of $\sim 12.5 \mathrm{~W} / \mathrm{nm}$ was achieved. The peak power density was increased to $26.9 \mathrm{~W} / \mathrm{nm}$ by reducing the repetition rate to $28 \mathrm{MHz}$. In Figure $7(\mathrm{~d})$ we plot data showing the pulse profile in the time domain at the fiber output which shows that the pulses remain relatively undistorted in the process.

\section{CONCLUSIONS}

We have reviewed some recent advances in the development of microstructured fibers for the delivery and generation of high power laser radiation. In particular we discuss loss/modality/bandwidth trade-offs for band gap fibers and speculate as to ultimate loss limits that may be possible by operating at longer wavelength, describe recent fabrication advances in the fabrication of shaped-core, high-NA JAC fibers and recent results in fibers for supercontinuum generation in the visible and mid-IR wavelength range. Our results highlight the unique and enabling fiber properties made possible by the microstructured approach and point to important future applications.

\section{ACKNOWLEDGEMENTS}

This work was funded in part by UK Technology Strategy Board grants LAMPS and PFIDEL and UK EPSRC grant EP/C515668/1. The authors would like to thank Dr T. Delmonte of BAe Systems, Prof. Howard Baker, Prof. Dennis Hall and of Herriot-Watt University and Dr Roy McBride PowerPhotonic Ltd. for useful discussions on diode-stack beam correction, beam-reformatting and coupling into optical fibers. In addition, collaboration with OpTek Ltd on the JAC fiber end-termination is gratefully acknowledged. Francesco Poletti and Jonathan Price gratefully acknowledge support from the Royal Society and the Royal Academy of Engineering Fellowships respectively.

\section{REFERENCES}

[1] R. Amezcua-Correa et al., Optics Express, Vol.14(17), 7974-7985, (2006).

[2] R. Amezcua-Correa et al., Proc. ECOC Cannes, (2006).

[3] R. Amezcua-Correa et al., Optics Express, Vol.16(2), 1142-1149, (2008).

[4] M.N. Petrovich et al., Optics Express, Vol.16(6), 4337-4346, (2008).

[5] P. J. Roberts et al., Optics Express, Vol.13(1), 236-244, (2005).

[6] J. K. Lyngso, et al., Proc. OECC'09, paper FS1, (2009).

[7] K. Imamura et al., Proc. OECC'09, paper FS5, (2009).

[8] K. Tajima, Proc. ECOC'07, Postdeadline paper PD2.1, (2007).

[9] J. Zhou et al., Optical Fiber Technology, 11, 101-110, (2005).

[10] C. J. Hensley et al., Optics Express, 15, 3507-3512, (2007).

[11] K. Furusawa et al., Optics Express, 9(13), 714-720, (2001).

[12] http://www.nktphotonics.com/side5322-cid-5215.html

[13] W. Wadsworth et al., IEEE Photonics Technology Letters, 16(3), 834-845, (2004).

[14] J.R. Hayes et al., Optics Express, Vol.14(22), 10345-10350, (2006).

[15] T. Delmonte et al., SPIE Conference: Optics/Photonics in Security, Stockholm, paper 6400-04, (2006).

[16] J.C. Flanagan et al., Optics Express, Vol. 16(23), 18631-18645, (2008).

[17] X. Feng et al., Optics Express, Vol.16(18), 13651-13655, (2008).

[18] A. Millo, L. Lobachinsky, A. Katzir, IEEE Photonics Technology Letters, Vol.20(9-12), 869-871, (2008).

[19] J.C. Flanagan et al., Optics Express, Vol.14(24), 11773-11786, (2006).

[20] J. Price et al., IEEE J. of Selected Topics in Quantum Electronics, Vol.13(3), 738-749, (2007).

[21] J. M. Dudley, G. Genty, and S. Coen, Reviews of Modern Physics, 78, 1135 (2006).

[22] J. M. Stone and J. C. Knight, Optics Express, Vol.16(4), 2670-2675 (2008).

[23] K.K. Chen et al., to appear Optics Express, (2010).

[24] J. C. Travers et al., Optics Express, Vol.16(19), 14435-14447 (2008). 